\title{
Two Distinct Fusiform Face Areas
}

\author{
Ahmad Yousef ${ }^{1}$ \\ ${ }^{1}$ School of Computational Science and Engineering, McMaster University, Hamilton, Ontario, Canada \\ *Correspondence: mohamas2@mcmaster.ca
}

\begin{abstract}
This article provides evidences that the fusiform face area connected to retinal peripheries trigger high graded face recognition; namely, the fusiform face area has two distinct areas, a central area and a peripheral one. We applied a tricky design to Rabbit-duck ambiguous image to approach this theory. We also provide evidence that the last point screened is responsible for the decisive face recognition. Introduction

The rabbit-duck illusion is an ambiguous image in which a rabbit or a duck can be accessed the human visual awareness. Fusiform face area is responsible for face recognition not only for human faces but animals too, therefore, it must have a role in understanding the neural correlates of the rabbit-duck illusion. Recent studies in face recognition had reported that; the brain activities in the FFA is greater for those faces
\end{abstract} closer to human face, such as the faces of cats and rabbits when compared with the faces of ducks and horses. Detailly speaking, human head produces MR signal much greater than the MR signal by horse head, (Kanwisher, etal. 1999), however, the faces of cats and humans shows equal brain activities; namely, 1.6\% MR BOLD signal, (Tong, etal. 2000). With perfect design, Jiedong Zhang, and his colleagues had recently been able to confirm that the FFA is engaged in holistic, but not parts-based, representation of faces, and this might give further explanation of why faces closer to human faces produce greater activities. Noticeably, except (Yaoda Xu, 2005), the aforementioned researches hadn't clearly compared which FFA is stronger, the right or the left. In 2012, Ming Meng and his colleagues, had suggested the left fusiform gyrus is responsible for graded analyses (quality of face recognition), but the right fusiform gyrus is responsible for categorization (whether there is a face). The current study, however, aims to test whether the central or peripheral retinal areas and their neurological connections to FFA have more responsibility for higher-grade face recognition, using a psychophysical experiment. For simplicity, we will call the two aforementioned different areas as follows: Central fusiform face area, central FFA, and peripheral FFA.

\section{Materials \& Methods}

Twelve right-handed human subjects were recruited to participate in this study. We used a renowned face rivalry stimulus that alternates the human visual awareness between duck face and rabbit face; see the central image. We firstly hypothesize that the differences between the two fusiform face areas are the reason of the rivalry; and therefore, we developed a novel technique to test this hypothesis. We simply apply motion on the aforementioned stimulus, and the background brightness has to change significantly. Namely, the stimulus moves from left to right for 40 seconds 'with a constant speed of 18 degrees/s'; and then moves from right to left for another 40 seconds. Two 'mirrored' stimuli had been tested for the purposes of counterbalancing. This design ensures accurate results because of the following points:

1- The applied motion will ensure presenting the stimulus to the both hemispheres, since the heads of the human subjects are placed centrally.

2- Increasing the brightness will ensure pupil constriction and therefore reduced access to the retinal peripheries.

3- Pursuit eye movements will emphasize the attention of human subjects, and therefore accurate reporting.

4- Pursuit eye movements will significantly minimize the spontaneous saccades and microsaccades; thus, their effects on face recognition can be omitted. Before we start the experiment, we make sure that all of the human subjects are able to notice face rivalry (duck- rabbit). Afterwards, we played the videos, see the supplement materials and references 10 , and 11 . Once the stimulus started to move, and just after the last point of the stimulus appears; each human subject has to report whether he/she saw duck or rabbit. In the second experiment, human subjects have to report whether the faces are switching (between duck and rabbit); and if yes, he/she has to report the location of switch, and the spatial locations of duck face, and rabbit face. The brightness of the screen will be varied to estimate whether pupil variations and the retinal peripheries play roles in face recognition. To maximize the effect of the brightness on pupil variation; we requested from the subjects to have coffee intake for the purpose of pupil dilation (see reference 13). The normalized values of the brightness will be 0 , and 1 , namely, 1 is the maximum brightness, and 0 is the minimum brightness. The distance between the screen and human subjects is 50 centimeters; and the stimulus height subtends 2.3 DVA, and its width subtends 3.77DVA.

Data Recording and Analysis

The data are collected manually using an installed meter over the screen. The data are afterwards analyzed, for each level of brightness, to obtain the mean value and the standard 
error of the mean for the twelve subjects. Regardless the direction of motion, all of the subjects had seen the duck in the left, and the rabbit in the right. Astonishingly, each human subject had reported the same switching location in their counterbalancing trials, but the spatial positions of the face were just mirrored. Namely, for the lowest brightness, the

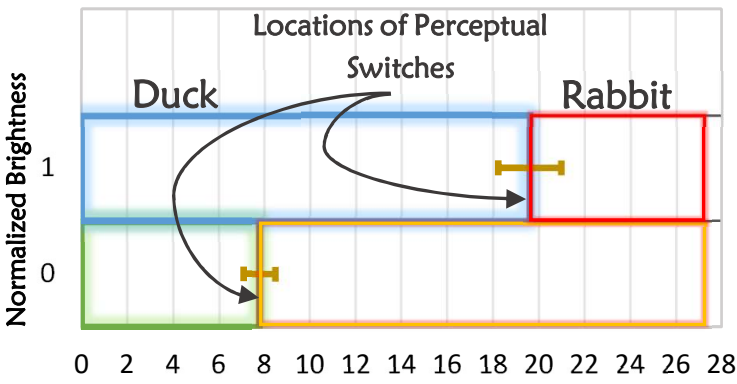
Perceptual Locations (degrees visual angle) accommodation, see reference 17; thus, the stimuli (faces) will be having greater accessibility to the retinal peripheries. Essential Notifications Readers should have direct access to the stimuli, see reference 10 , and 11. Advisably, download the video for the best quality. For validation process, stop the video in whatever time you want, you may notice rivalry between duck and rabbit face rabbit face subtended larger visual space, but, in the left visual space instead of the right. The variation of pupil size therefore played a significant role in the switching position, in another word, lower brightness, and it corresponding larger pupil allows the rabbit to subtend great visual space, as seen in the central figure.

\section{Discussion and Conclusion}

The current research concludes that the last point screened in the most important point to the final decision of face recognition, which means that face recognition is a hierarchical neural process. The results also clearly concluded the following; the 'peripheral FFA' is responsible for creating high-grade face recognition, but the 'central FFA' is responsible for low-grade ones. We believe that for high grade face recognition, the peripheral and the central FFA do work collaboratively, but for lower grade ones, only the central is activated; this consistent with the fact that higher graded face recognition produced higher MRI signals. Based on the current results, we do disagree with Ming Meng and his colleagues conclusion; namely, we think that both fusiform gyrus, the left and the right, are responsible for graded analyses namely the perception of higher order faces (rabbit), as well as, the categorization. The real challenge is to distinguish between the brain regions in the FFA which neurologically and exclusively connected to the retinal peripheries or the central retina. Important to mention, pupil dilation might accommodate the lens (see reference 7) and therefore the light rays will feed greater areas of retinal peripheries, namely, faster signals to the FFA, which causes faster recognition of the rabbit face. However, pupil constriction tends to flatten the lens, namely, weaker accessibility to retinal peripheries, and therefore the recognition of rabbit face will be harder (see references 8 , and 9 for better comprehension of how the retina signals the brain). Pupil constriction is common among elderly people (see reference 14), and this reason might be the main reason of the degradation of face recognition (see reference 15); greater contrast could dilate pupil size, see reference 16 , thus, it should result in better face recognition for those with constricted pupils. We therefore highly recommend for elder adults to have daily intake of natural supplements that can dilate the pupil to improve their ability in face recognition. In fact, caffeine intake also dilate the pupil and enhances for the stationary stimulation. To validate the concept of the two FFA, change your screen brightness, namely, try maximum brightness (pupil constriction, weaker access to retinal peripheries, and thus, lower graded analysis for face recognition) versus the minimum brightness (pupil dilation, greater access to retinal peripheries, and thus, greater graded analysis for face recognition, namely, faces closer to human faces 'rabbit face' will be easily recognized, and dominate). Readers should connect the author directly in case of they cannot access the stimuli. Scholars with brain imaging facilitations are welcome to collaborate in the aforementioned challenging investigations. Enquiries should be sent to the author.

\section{Transactional References}

[1] Weisstein. "Rabbit-duck illusion". MathWorld.

[2] Kanwisher, etal. 1999. "The fusiform face area is selective for faces not animals". Neuroreport.

[3] Tong, etal. 2000."Response properties of the human fusiform face area". Cognitive Neuropsychology.

[4] Zhang, etal. 2012. "The Fusiform Face Area Is Engaged in Holistic, Not Parts- Based, Representation of Faces". POLS One.

[5] Yaoda Xu, 2005. "Revisiting the Role of the Fusiform Face Area in Visual Expertise". Cerebral Cortex.

[6] Meng, etal. 2012. "Lateralization of face processing in the human brain". Proc Biol Sci.

[7] Yousef, Ahmad. 2019. "Linking Dynamics Between Pupil and Lens." engrXiv. doi:10.31224/osf.io/9c3vk.

[8] Masland, (2017). Vision: Two Speeds in the Retina. Current Biology,

[9] Yousef, Ahmad. 2019. "Consciousness Might Be Localized in Extra Physical Dimensions." PsyArXiv. 2019. doi:10.31234/osf.io/angc8.

[10] The moving stimuli Left $\rightarrow$ Right may be found here:

https://drive.google.com/drive/folders/1GHZdTHnxjBWb6r514t9GYI34d7 nxcgEw? usp = sharing

[11] The moving stimuli Right $\rightarrow$ Left may be found here: https://drive.google.com/drive/folders/1q3rO6Ny_CQFLLg1Oy6BoqEFa 9cBLuf-?usp=sharing

[13] Abokyi, etal.,(2017). Caffeine intake is associated with pupil dilation and enhanced accommodation. Eye (Lond) Journal.

[14] Sloane, etal. (1988). Aging and luminance-adaptation effects on spatial contrast sensitivity. J Opt Soc Am A.

[15] Boutet, etal. (2015). On the particular vulnerability of face recognition to aging: a review of three hypotheses. Frontiers in psychology.

[16] Wang etal. (2014) Modulation of stimulus contrast on the human pupil orienting response, in European Journal of Neuroscience.

[17] Abokyi, 2017. Caffeine intake associated with pupil dilation \& enhanced accommodation. Eye Journal. 